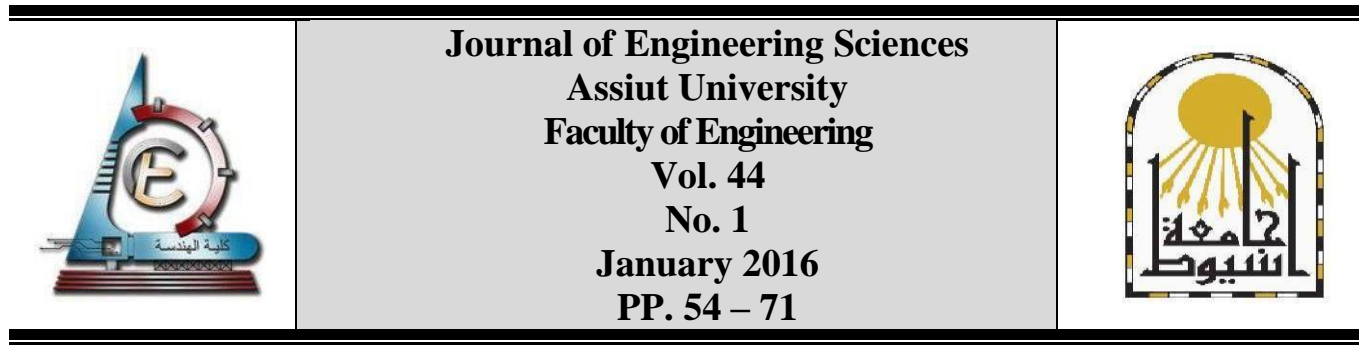

\title{
DEVELOPMENT AND VALIDATION OF AN IN-HOUSE CFD CODE FOR ENVIRONMENTAL FLOW SIMULATION USING MEASUREMENTS IN ATMOSPHERIC BOUNDARY LAYER WIND TUNNEL
}

\author{
Walid J. Al-Nahari, Mohammed F. F. El-Dosoky, \\ Mohammed M. Abdelghany, and Hamdy M. Shafey \\ Mechanical Eng. Dept., Faculty of Engineering, Assiut University, Assiut, Egypt
}

Received 15 December 2015; Accepted 15 January 2016

\begin{abstract}
This paper deals with the important issue of the gaseous pollutants dispersion in the environmental flow within urban atmospheres including the products of on-ground agriculture fires. The paper presents an accurate and efficient semi-implicit pressure-based algorithm developed for solving numerically the conservation equations governing weakly compressible turbulent environmental flow of multicomponent fluid. An in-house CFD code has been developed to implement the numerical solution of the present algorithm. The code is an integrated one consisting of three main elements which are the pre-processor, the solver and the post-processor. This in-house CFD code undergoes a standard validation process, using six generic test cases simulating a steadystate environmental flow associated with gas flow from a ground point source under different flow conditions. A prerequisite verification of the code has been considered for successful validation. The detailed standard validation process was performed according to AIAA Guide by comparing the predicted results with measurements especially conducted for the present work in the test section of the Atmospheric Boundary Layer Wind Tunnel facility of Assiut University. The results showed good qualitative and quantitative agreements for the present in-house CFD code with the corresponding flow measurements. These agreements together with the satisfaction of the prerequisite verification insure a satisfactory validation of the present in-house CFD code.
\end{abstract}

Keywords: Environmental Flow, Atmospheric Boundary Layer Wind Tunnel, In-House CFD code, Simulation, pollutants dispersion, verification and validation.

\section{Introduction}

Environmental flow studies investigating pollutants transport and dispersion in urban atmospheres are critical for air quality control. This is mainly because industrial enterprises, transportation means, and other sources of air pollution are frequently located within urban areas. In addition to the mentioned sources, agricultural fires occurring in open spaces of urban areas produce more air pollution (thermal, gaseous and particulates). The majority of pollutants emitted from sources of air pollution in urban areas are released into the part of the atmosphere adjacent to the earth's surface. This part is known as the atmospheric boundary layer which has a typical height of 500-600 $\mathrm{m}[1,2]$. The environmental flow is usually 
characterized by a turbulent weakly compressible flow occurring in the atmospheric boundary layer. It also involves physical complexity due to the link and interaction between dispersion of the pollutants and other transport phenomena. More geometrical complexity is added due to buildings with different shapes and heights forming narrow and deep street canyons which are distributed over the urban area.

Theoretical and experimental research tools [2-10] were introduced to handle the environmental flow problems. Successful research tools are expected to give accurate information on the near-ground concentrations of air pollutants. Their ability to achieve such a task depends on how can they accurately and correctly describe the real and complex situation of the environmental flow. The major and most successful theoretical tool giving accurate numerical predictions is the commonly used approach of computational fluid dynamics (CFD) simulations. The CFD approach numerically solves the appropriate fluid flow equations governing various processes and events leading to the pollutants dispersion. Successful and frequently done laboratory measurements, supporting the CFD simulations, are those conducted in atmospheric boundary layer wind tunnels facilities (ABLWT) [7-10]. Such facilities allow accurate physical modeling of real environmental flow within atmospheric boundary layer for specified urban area. The design and characterization of ABLWT have received the attention of many research workers in the last three decades [5-9, 11-15].

An important issue is necessary to establish and assess the credibility of newly developed CFD codes. This issue is known as verification and validation (V\&V) processes. Many professional engineering organizations and societies introduced comprehensive reviews and recommended broad publications [16-21] related to $\mathrm{V} \& \mathrm{~V}$ processes. One important publication by the American Institute of Aeronautics and Astronautics is the AIAA Guide [16] which includes useful and integrated guidelines for the terminology and methodology for standard V\&V processes. Many studies [22-28] have applied the AIAA Guide methodology, to investigate the validation of different CFD codes through the comparison of their predictions with the corresponding experimental results representing the real environmental flow. Most of these experimental results were obtained from measurements best conducted in ABLWT facilities.

Recently, the authors [29] have developed an integrated in-house CFD code to implement the semi-implicit pressure-based algorithm they proposed for simulating singlecomponent fluid flow. Focusing on the standard verification process they applied the principles of the AIAA Guide and reached a satisfactory verification (code correctness and calculation accuracy) of their in-house CFD code. This was done by comparing their code numerical predictions with benchmark solutions of reference CFD codes [30-34] for selected standard test cases. They concluded that their code is able to predict various flow features in the selected test cases in agreement with the reference codes. Accordingly, they simply reported that this agreement supports the validation of their in-house CFD code. However, the algorithm presented in [29] needs more development to include more features of environmental flow in urban areas. In addition, standard validation process of the new resulting in-house CFD code must be carried out according to the AIAA Guide.

The aim of the present paper is to model and solve the environmental flow involving gaseous pollutants dispersion in urban atmospheres. This is done by developing the solution algorithm described by the authors in [29], through the inclusion of mass species 
Walid J. Al-Nahari et al., Development and validation of an in-house cfd code for ..............

conservation equations governing the pollutants dispersion. Moreover, the previous inhouse CFD code presented in [29] is to be modified to implement the developed solution algorithm of the environmental flow. This newly in-house CFD code undergoes a standard verification and validation processes using six generic test cases. These test cases have been generated by imposing three different flow conditions on two physical model configurations. The standard verification process, which is a prerequisite for the required standard validation process, has been performed by comparing the predictions of the present in-house CFD code with the corresponding predictions of a reference CFD code, ANSYS Fluent 16. On the other hand, the standard validation process has been performed according to AIAA Guide by comparing the predictions of the present in-house CFD code with the corresponding measurements especially conducted for this purpose in the test section of the ABLWT facility of Assiut University. The wind tunnel facility enables measuring the distributions of the average velocity, turbulence intensity, temperature, and tracer gas concentration of the wind flow. These measurements have been performed at the inlet and at different locations within the test section. The inflow conditions were taken from the measurements in order to correctly reproduce the flow into the computational domain for both the present in-house CFD code and the reference code.

\section{The Computational fluid dynamics (CFD) model}

\subsection{The present numerical algorithm}

The present algorithm is a semi-implicit pressure-based one. It is an extension of the algorithm developed previously by the authors [29] to numerically solve the Navier-Stokes and energy equations governing weakly compressible flow of a single component fluid. The present algorithm numerically solves the mass species conservation equations in addition to other governing equations for weakly compressible turbulent environmental flow of multicomponent fluid. This fluid flow can be described by the time dependent three dimensional Favre-averaged conservation equations combined with the standard $k$ - $\varepsilon$ turbulence sub-model equations. These fluid flow equations can be represented in the following general form [35]:

$$
\frac{\partial(\rho \phi)}{\partial t}+\nabla \cdot(\rho \vec{U} \phi)=\nabla \cdot\left(\Gamma_{\phi} \nabla \phi\right)+S_{\phi},
$$

where $\vec{U}$ is the vector of the average flow velocity, and $\phi$ is the generic form of the transport fluid property. Table 1 of [29] lists the expressions of the generic form, $\phi$, and associated diffusive exchange coefficient, $\Gamma_{\phi}$ and source term $S_{\phi}$, for momentum and energy transport fluid properties. On the other hand, the expressions for mass transport fluid properties (in absence of mass production) are given in [35] by:

$$
\begin{aligned}
& \phi=Y_{m i} \\
& \Gamma_{\phi}=\frac{\mu_{e}}{\sigma_{Y_{m i}}} \quad(i=1,2, \ldots, n) \\
& S_{\phi}=0
\end{aligned}
$$$$
\}
$$

where $Y_{m i}$ is the species mass fraction of component $i$ in the fluid of $\mathrm{n}$ components, and $\sigma_{Y_{m i}}$ is the corresponding Prandtl number/schimdt number. The effective turbulent viscosity, 
$\mu_{e}$, is the sum of the molecular dynamic viscosity, $\mu$, and turbulent viscosity, $\mu_{t}$, expressed in terms of the turbulence transport fluid properties $k$ and $\varepsilon$ as given in details in [35]. In writing the expressions of Eq. (2) the flow conditions of the common practical applications of environmental flow have been considered such that only the ordinary (concentration) diffusion is included while the pressure, forced and thermal diffusions are neglected [36].

The numerical solution for the general case of a time dependent flow field in a geometrically complex space domain (computational domain), has the following main features. The time domain is discretized using suitable time steps while the whole computational domain is first partitioned into multi-blocks sub-domains. Then, the computational sub-domain associated with each block is by turn discretized into structured non-orthogonal boundary-fitted curvilinear mesh of cells of finite volumes. The numerical values of the transport fluid properties describing the discretized flow field are obtained for each time step by iteratively solving a system of linear algebraic difference equations approximating the fluid flow. The algebraic difference equations are obtained for each transport fluid property at the specified discrete space points (centers of interior cells) by substituting in the discrete version of the fluid flow equations combined with the discrete form of the boundary conditions. These conditions mainly include the fixed conditions imposed on the physical boundaries of the whole computational domain (inflow, outflow, solid wall, far-field, and symmetry). In addition, they include the connectivity conditions imposed at the interfaces between the blocks. The discrete version of the fluid flow equations is obtained by applying the Gauss divergence theorem after integration over individual timeinvariant finite volumes of the interior cells covering the whole computational domain.

\subsection{The present in-house CFD code}

The present in-house CFD code implementing the above described steps of the present algorithm is a modification of the previously developed one [29]. It consists of three main elements: the pre-processor, the flow solver, and the post-processor. The pre-processor transforms the input data and information into a suitable form. The flow solver handles the transformed input data and fulfills the steps of the numerical solution of the present algorithm. The post-processor receives the output of the flow solver in the form of numerical predictions and presents it using versatile data visualization tools.

The flow solver of the present in-house CFD code mathematically solves the equations of fluid flow associated with numerous physical phenomena which are characterized with a variety of thermophysical properties. It generally handles different geometries of inner and outer boundaries, combined with various initial and boundary conditions. This description shows two important features of the code. The first feature is that the code numerically simulates the fluid flow through a series of complex mathematical activities. Consequently, both the correctness of the code mathematical activities and the accuracy of its calculations must be verified. The second feature is that the physical phenomena and the thermophysical properties considered in the real fluid flow are, in general, complex in nature and are governed with known and well-established physical laws. Accordingly, the code ability to physically handle real flow situation must be validated.

\subsection{Verification and validation processes}

For acceptable credibility level of the present in-house CFD Code, standard verification and validation processes have been performed according to AIAA Guide [16], using different 
Walid J. Al-Nahari et al., Development and validation of an in-house cfd code for ..............

test cases. The standard verification process of the present in-house CFD code including both code correctness and calculation accuracy verifications represents a prerequisite for the standard validation process which is one of the main objectives of the present work. Both code correctness and calculation accuracy verifications were partly confirmed for the previous in-house CFD code [29] through comparison with benchmark solutions of willestablished reference CFD codes [30-34]. They are to be completed upon the discussion on the comparison of the present in-house CFD code predictions with the corresponding predictions of another reference CFD code, ANSYS Fluent 16. On the other hand, the validation of the in-house CFD code is recognized upon the discussion on the comparison of its predictions with the corresponding measurements. These measurements were conducted in the test section of an ABLWT facility whose details are outlined in the next section.

\section{The Experimental facility of the atmospheric boundary layer wind tunnel (ABLWT)}

The measurements for the validation of the present in-house CFD code, were performed in the ABLWT facility located in the laboratory of Environmental Studies and Research at Assiut University. The following subsections summaries the main features of the wind tunnel, the associated instrumentation and measuring devices, and the used test physical model.

\subsection{Description of the ABLWT}

The ABLWT used in the present work, was previously, designed, constructed, and characterized to investigate environmental turbulent flow studies [1,2]. Figure 1 shows the main features of the ABLWT which is of a low-speed, open-loop type. Dust-free clean atmospheric air enters the wind tunnel smoothly through the upstream settling section $\mathbf{1}$ and is accelerated in the contraction cone 2. The air then flows through a heating and settling section 3 to produce a thermal stratification in the air flow (if required). The resulting uniform turbulent air flow passes in the development section $\mathbf{4}$ to produce a wind with the required simulated atmospheric boundary layer. This is done via a combination of triangular spires and cube roughness elements [37] designed to artificially generate the earth surface condition associated with urban areas. The test section $\mathbf{5}$, in which the test physical model is mounted, is $1.7 \mathrm{~m}$ long and has a square cross section of $1.0 \mathrm{~m} \mathrm{X} 1.0 \mathrm{~m}$. It receives the longitudinal turbulent wind flow whose average velocity distribution is described by a power law. The wind tunnel is driven by an axial flow fan $\mathbf{7}$ which is operated by a variable speed motor giving a maximum operating wind velocity of $2 \mathrm{~m} / \mathrm{s}$. A flexible connection 6 is used to isolate the fan vibrations from the main body of the wind tunnel.

\subsection{Main instrumentation and measuring devices}

A variable voltage transformer has been used with a three phase power supply to adjust the fan motor speed at a value corresponding to a prescribed wind flow velocity profile. The intended single measuring probe or sensor is mounted on a 3D traversing mechanism which enables conducting measurements at different specified locations covering the space in the test section. A tracer gas (CO2) bottle and a compressed air supply have been used with a gas flow controller and an electric air heater for supplying and controlling cold or hot jet of $\mathrm{CO} 2$ or air in the measurements of the test cases in the present study. The gas flow controller has been used to set a measured value of flow rate corresponding to a prescribed mean gas velocity. A computer-linked Multi-Channel Hot-wire Anemometer has been used to measure the magnitude of local average wind velocity and associate turbulence intensity in the test section. The anemometer is provided with two spherical probes for the velocity vector and 
two directional probes for the velocity components. In addition to wind velocity, the anemometer can be used to measure the relative humidity and the wind temperature up to 70 ${ }^{\circ} \mathrm{C}$. Other temperature measurements have been performed using Type $\mathrm{K}$ thermocouples with Data Acquisition System. An automotive flue gas analyzer has been used to measure the volume fraction of the tracer gas in the test section. The measured gas volume fraction is a representative of the local gas concentration.

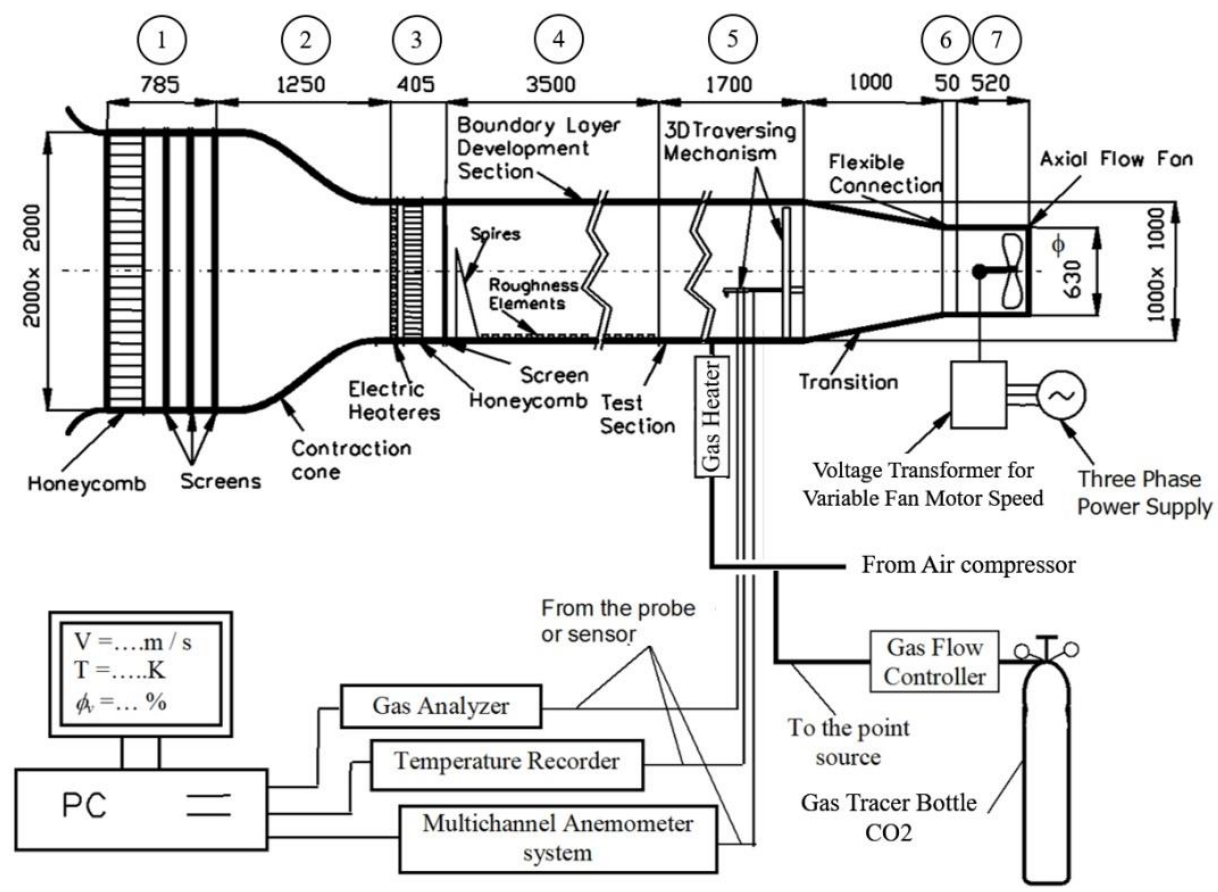

Fig. 1. Main features, instrumentation, and measuring devices of the ABLWT (dimensions in mm).

\section{The Test cases}

Six generic test cases are considered in the present work for both standard verification and validation processes. Each test case is a simple simulation of a steady-state environmental flow associated with gas flow from a ground point source in the urban area. Examples of ground point sources are the upward air flow from the ventilation openings of subways and underground cities as well as the upward flow of gaseous products from a surface agricultural fire within limited zone. Figure 2 shows the conceptual model illustrating the environmental flow simulated by a generic test case. The conceptual model comprises four main components: the CFD computational domain, the inflow conditions, the gas flow from the point source, and the existence or absence of a nearby building. The choice of the generic test cases is based on the following considerations for the selection of each component. The open field full-scale CFD computational domain may lead to inaccurate correspondence with the measurements conducted in the closed test section of the ABLWT facility. Therefore, the CFD computational domain is selected to have the boundaries and cross-section dimensions $(W c=1 \mathrm{~m}, H c=1 \mathrm{~m})$ of the test section. Accordingly, the inflow boundary conditions can be prescribed based on the measurements of the steady-state longitudinal turbulent wind flow at the inlet of the test section. The 
Walid J. Al-Nahari et al., Development and validation of an in-house cfd code for ..............

major vertical variation of the measured average velocity of the wind flow $($ at $x=0)$ is typically described by the following power law function:

$$
u(z)=u_{\delta}(z / \delta)^{\alpha},
$$

where $\delta$ and $\alpha$ are the simulated atmospheric boundary layer thickness and the associated exponent for the urban area, respectively. The values of $\delta$ and $\alpha$ were determined from curve fitting of measured velocity distribution to be $0.51 \mathrm{~m}$ and 0.32 , respectively. The outflow boundary of the CFD computational domain is apart from the inflow boundary by a distance $L c$ which varies according the physical model configuration as specified in the next section. The gas flow from the ground point source with a circular cross section is represented by an upward gas jet located at $l_{j}=0.2 \mathrm{~m}$ from the inlet and centered in the $y$-direction of the test section. The gas jet is in steady-state crossflow relative to the longitudinal wind. The upward velocity distribution around the point source within the jet, $w(x, y)$ is obtained using the measured gas flow rate. The velocity $w(x, y)$ is estimated considering laminar flow out from a circular tube of a diameter $d=7.6 \mathrm{~mm}$, centered at the point source. The nearby building (if exists) is simulated by a cubic-shaped block with edge length $l=0.2 \mathrm{~m}$. The block is centered in the y-direction in the test section with its windward wall located downstream at a distance $l_{b}=0.22 \mathrm{~m}$ from the test section inlet. The windward wall can be uniformly heated to reach a steady state temperature $T_{w}$. This can be done using an electric heater assembly buried inside the block. The heated windward wall represents the real condition associated with the building heating sources (solar irradiation, ovens, and compartment fires). To complete the choice of the test cases, two possible physical model configurations have been produced. They are a free gas jet (configuration A), and a block with adjacent gas jet (configuration B). Figure 3 shows the main dimensions of the two model configurations with selected locations at each one of which the vertical distributions of the main transport flow properties have been measured. Finally, six generic test cases can be generated by exposing each produced configuration to three possible different flow conditions. Keeping in mind the common cold inflow conditions, the chosen test cases can be briefly identified as:

1. Cold air jet.

2. Hot air jet.

3. Cold pollutant gas (CO2) jet.

4. Cold air jet adjacent to a block with cold walls.

5. Cold pollutant gas (CO2) jet adjacent to a block with cold walls.

6. Cold pollutant gas ( $\mathrm{CO} 2)$ jet adjacent to a block with heated windward wall. 


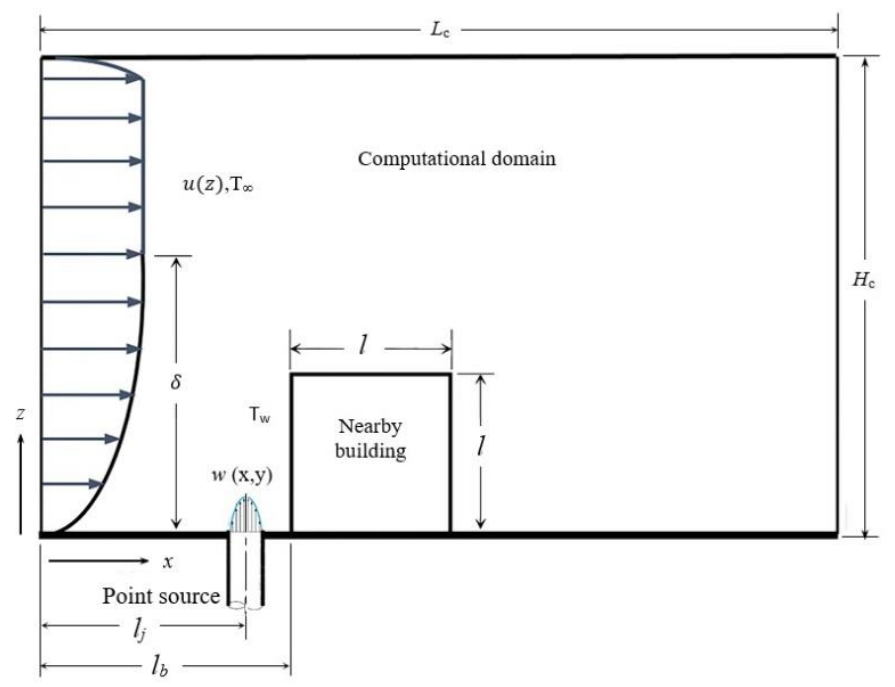

Fig. 2. Components of the conceptual model for the generic test cases.

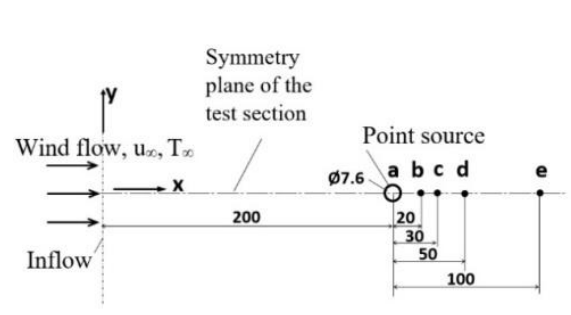

Configuration A

[measurements locations: a (center of the gas

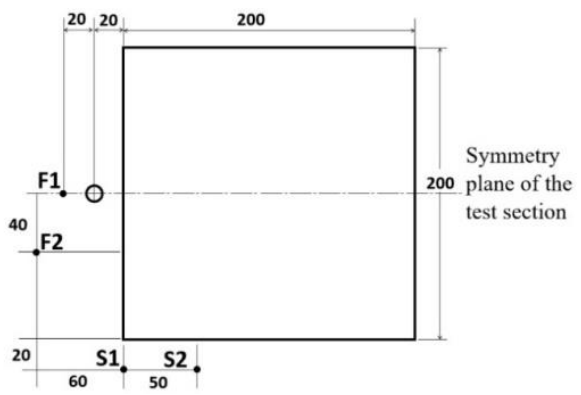

ource),b,c,d and e]

Fig. 3. Top view of the physical model configurations showing the selected measuring locations. (dimensions in $\mathrm{mm}$ )

\section{Results and discussions}

In conducting the experiments, the operation of the ABLWT properly controlled to obtain steady wind flow, with low level of the noise (internal and external) that might affect the flow stability and consequently the measurements. In all test cases, the wind flow was adjusted to give a fixed free stream velocity $u_{\delta}=0.5 \mathrm{~m} / \mathrm{s}$. The volume flow rate of the gas jet was adjusted to give a fixed maximum source velocity of $2.24 \mathrm{~m} / \mathrm{s}$ at the jet center for cold and hot conditions. The measured flow characteristics presented and discussed below have been chosen to give representative experimental results for the validation process. Selected measured transport properties for each test case were considered for the comparison with the CFD predictions. The assumption of the outflow boundary of the CFD computational domain was checked via calculations using different values of the length $L c$. The calculations showed appropriate $L c$ values of $0.5 \mathrm{~m}$ and $1.02 \mathrm{~m}$ for the physical model configurations A and B, respectively. These values of $L c$ with the other specified dimensions 
Walid J. Al-Nahari et al., Development and validation of an in-house cfd code for ..............

( $W c=1 \mathrm{~m}, H c=1 \mathrm{~m}$ ) were adopted as the best values to obtain all numerical predictions of both the in-house and the reference CFD codes.

The discussion of the obtained experimental and numerical results is carried out in three steps. The first step involves the comparisons between the predictions of both the in-house and the reference CFD codes, along with the comparisons between the predictions of the present in-house CFD code and the flow measurements. The second step is the estimation of the code prediction and experimental errors. The third step focuses on the standard validation process for the present in-house CFD code. In this process the satisfaction of the verification prerequisite condition for the present in-house CFD code is examined based on the discussion in the previous steps. Then the validation for the present in-house CFD code is examined via the quantitative and qualitative comparisons with the flow measurements. For convenience, the comparisons in the first step of the discussion are made considering four integrated groups of flow property variations. Two groups expressing the flow characteristics which are the variations of the average flow velocity and the turbulence intensity. The third group expressing the flow thermal behavior is the variations of flow temperature. The forth group expressing the pollutant gas dispersion is the pollutant gas (CO2) concentration. The predictions for the $\mathrm{CO} 2$ gas concentration are represented by the plots of $\mathrm{CO} 2$ volume fraction $\mathrm{Y}_{\mathrm{v}, \mathrm{CO} 2}$. The values of the volume fraction were calculated using the corresponding values of the mass fraction $\mathrm{Y}_{\mathrm{m}, \mathrm{CO} 2}$ as follows:

$$
Y_{v, \mathrm{CO} 2}=\frac{\left(Y_{\mathrm{m}_{\mathrm{CO}}} / \mathrm{M}_{\mathrm{CO}_{2}}\right)}{\sum_{i}\left(Y_{m, \mathrm{CO}_{2}} / M_{i}\right)}
$$

where $M_{i}$ is the Molecular weight of the component $i$.

\subsection{Flow characteristics}

The two main flow characteristics for all test cases are the vertical variations of the average flow velocity components and the turbulence intensity. Such variations strongly influence the pollutant gas dispersion, compared with the variations of other flow properties. Figure 4 shows the results of a representative group for the velocity components vertical distributions. The results include the longitudinal $(\mathrm{u})$ and vertical $(\mathrm{w})$ velocity components for the flow in configuration $\mathrm{A}$, and that for the distributions of the total value $(\mathrm{U})$ of the average flow velocity for the flow in configuration B. Figure 5 shows the results of the corresponding vertical distributions of turbulence intensity.

The numerical predictions of the present in-house CFD code shown in Figs. 4 and 5 are in good qualitative and quantitative agreement with the corresponding predictions of the reference CFD code, ANSYS Fluent 16. The agreement can be understood from the identical trends (shape, size and location) of the vertical variation of the average flow velocity predictions of both the in-house and the reference CFD codes as shown in Fig. 4a. Similarity of these trends are clearly observed in the distortion of the inflow characterized by the features of the u component velocity profiles, combined with the production of the vertical component velocity profiles $\mathrm{w}$ associated with the upward flow of the free gas jet. The agreement can be supported by comparing the predictions of the vertical variation in the total flow velocity $U$ for both codes for configuration B, Fig. 4b. More evidence of the agreement between the two codes can also be obtained by comparing their predictions for the turbulence intensity shown in Fig. 5. The results in Figs. 4 and 5 show noticeable differences at low levels above the ground wall corresponding to the gas jet levels. These 
differences may be attributed to the more accurate near-wall-treatment (as expected) of turbulence and fine mesh scheme used in the reference CFD code.

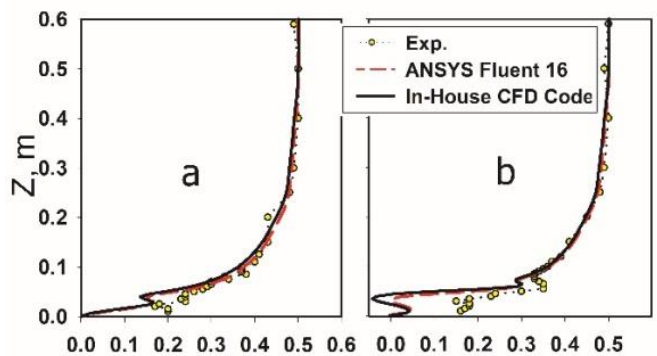

$\mathrm{u}, \mathrm{m} / \mathrm{s}$

$\mathrm{u}, \mathrm{m} / \mathrm{s}$

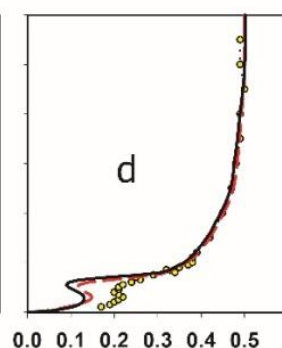

$\mathrm{u}, \mathrm{m} / \mathrm{s}$

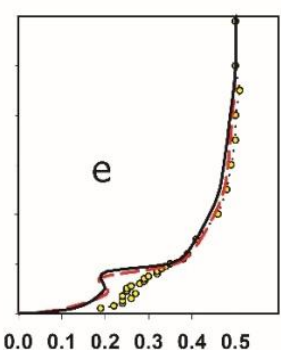

$\mathrm{u}, \mathrm{m} / \mathrm{s}$

i- longitudinal velocity component
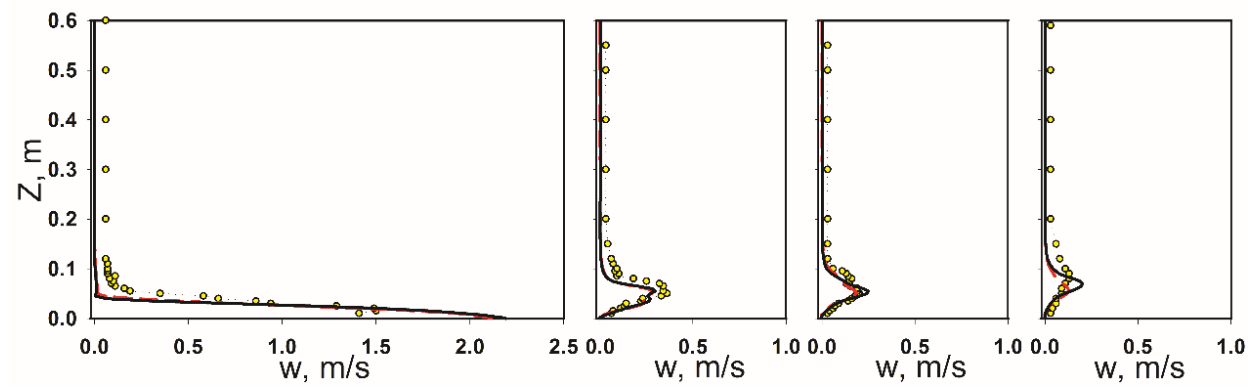

ii- vertical velocity component

Fig. 4. a. Vertical distributions of the longitudinal and vertical flow velocity components in the symmetry plane at different locations for configuration A.
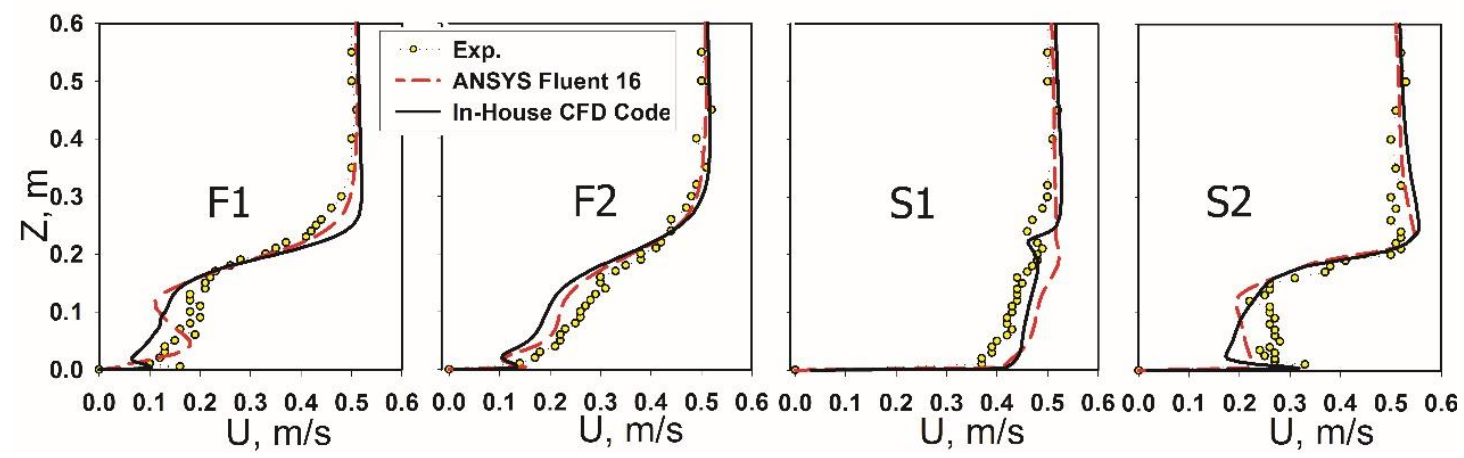

Fig. 4. b. Vertical distributions of the total average flow velocity $U$ at different locations for configuration $\mathrm{B}$.

The predictions of the in-house CFD code agree well with the velocity and turbulence intensity measurements in Figs. 4 and 5, particularly at higher levels above the jet in configuration A and at levels above the block height in configuration B. At these levels the predictions and measurements show nearly the same trends. Below these levels, there are slight discrepancies between the in-house CFD code predictions and excremental measurements of both the velocity and turbulence intensity. This is mainly due to the 
experimental errors associated with the disturbance introduced at low levels near the ground due to the finite size of the velocity sensing probe. On the other hand, the predictions exhibit relatively larger values of the turbulence intensity at such low levels. This can be also interpreted by the combined effects of the experimental errors and the less accurate treatment of near-wall turbulence in the in-house CFD code.
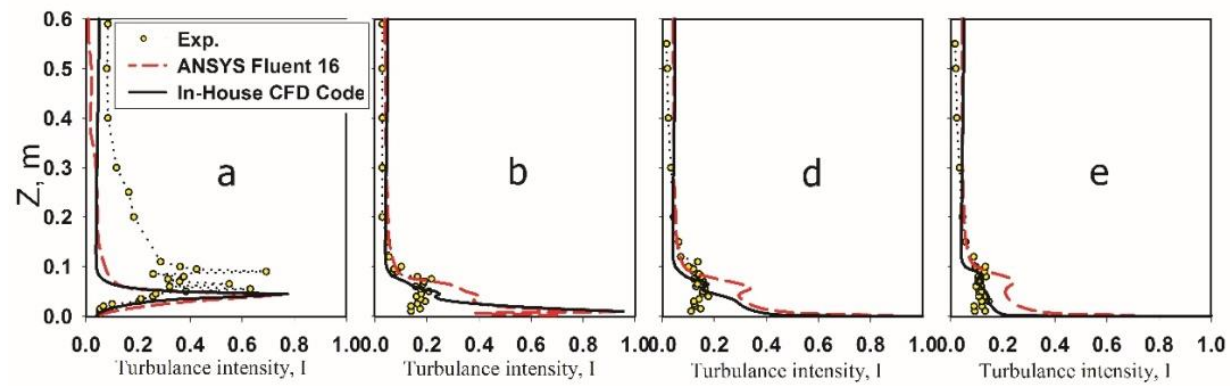

Fig. 5. a. Vertical distributions of the turbulence intensity in the symmetry plane at different locations for configuration A

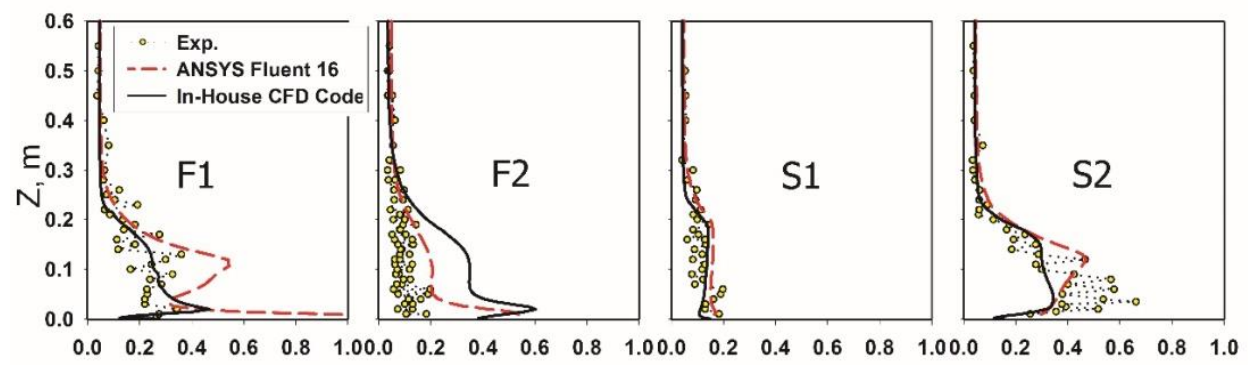

Fig. 5. b. Vertical distributions of the turbulence intensity in the symmetry plane at different locations for configuration $\mathrm{B}$

\subsection{Flow thermal behavior}

The results shown in Fig. 6 constitute a simple representative group for the temperature vertical distributions of the flow associated with the free hot air jet. A similar discussion as for the flow velocity can be made for the good agreement between the temperature predictions of the in-house CFD code and the corresponding predictions of the reference CFD code. The agreement for the temperature distributions introduces more evidence for the velocity distributions due to the link between the energy and momentum equations. Investigating the measured flow temperature distributions, one can clearly observe the continuous development in the shape, location, and beak of the hot jet temperature profile downstream of the wind flow. The results indicate that these observations are well reproduced by the in-house CFD code. 

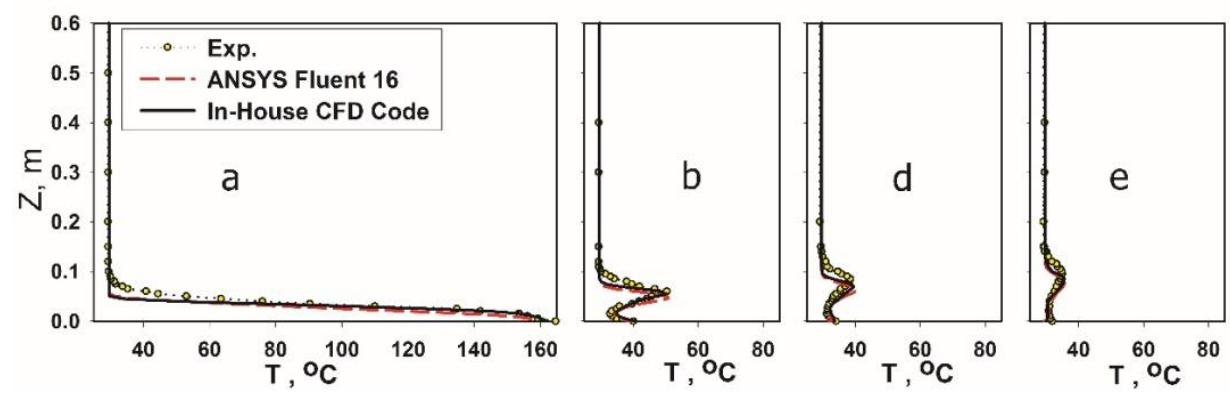

Fig. 6. Vertical distributions of the flow temperature in the symmetry plane for configuration A associated with the free hot air jet

\subsection{Pollutant gas dispersion}

Figure 7 shows the results of a representative group of vertical distributions of $\mathrm{CO} 2$ volume fraction for the two configurations exposed to different thermal conditions. The results in Fig.7a show the important features of the volume fraction vertical distributions downstream the free jet of cold $\mathrm{CO} 2$ for configuration A. The distributions are nearly symmetric, each around an axis inclined in the dominant direction of the wind velocity given by the $u$ and $w$ components shown in Fig. 4. The beak value of the volume fraction decreases as the location goes far downstream the $\mathrm{CO} 2$ jet. These features are direct results for the combined pollutant dispersion processes of the convective and diffusion mass transfer. Practically in environmental studies and its applications, it is usually required to predict the volume fraction of a pollutant at any space point downstream the pollutant gas flow from a ground source exists at certain location in urban area. The common form of the volume fraction plots in Fig.7a makes this task possible. This form can be nearly fitted with the known generalized Gaussian dispersion model [37]. It is a mathematical expression that gives the value of the pollutant volume fraction at a specified space point in terms of the magnitude and location of the peak value.

The results in Fig.7b and c illustrate the behavior of the pollutant gas dispersion linked with the typical characteristics of the cross wind flow around the block in existence of the upward flow of the gas jet. This behavior can be noticed by examining the vertical distributions of $\mathrm{CO} 2$ volume fraction at locations F1, F2, S1, S2 (Fig. 3) representing regions upstream and aside the cold windward wall of the block, Fig. 7b. The near ground smaller values of the $\mathrm{CO} 2$ volume fraction at location $\mathrm{F} 1$ are mainly due to the dominant effects of eddies and separation in the upstream region of the block. On the other hand, these effects are negligible at location F2 while the mass diffusion is dominant resulting in near ground larger values of the $\mathrm{CO} 2$ volume fraction as shown in Fig 7b. Remarkable values of the $\mathrm{CO} 2$ volume fraction are observed at far locations S1 and S2 adjacent to the side wall of the block. These observations may be attributed to a dominant mass diffusion at these locations with nearly absence of eddies and flow separation there. Practically, the results shown at the locations $\mathrm{S} 1$ and $\mathrm{S} 2$ indicate that the gaseous pollutant emerging from a ground source near a building can be appreciably sensed even at region close to the side of the building. 

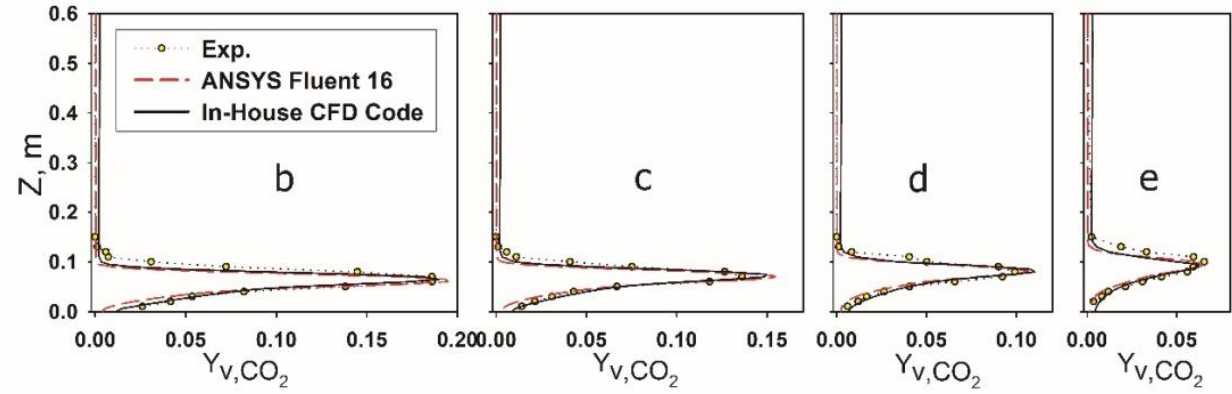

Fig. 7. a. Vertical distributions of the $\mathrm{CO} 2$ volume fraction in the symmetry plane for configuration A.
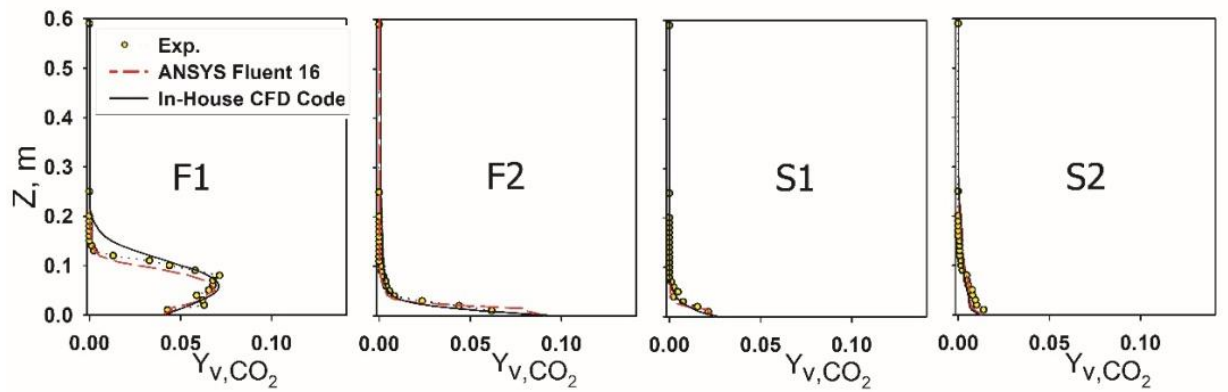

Fig. 7. b. Vertical distributions of the $\mathrm{CO} 2$ volume fraction in the symmetry plane for configuration $\mathrm{B}$ with the cold windward wall.
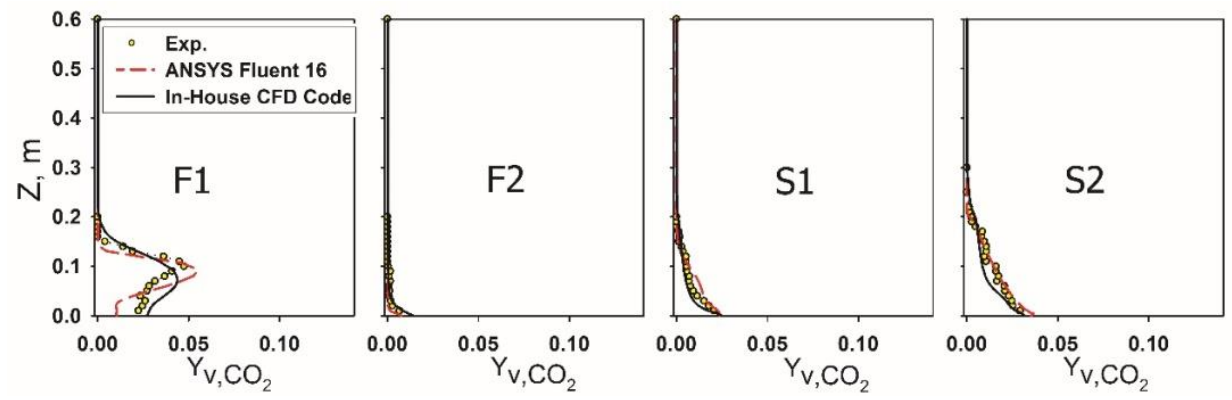

Fig. 7. c. Vertical distributions of the $\mathrm{CO} 2$ volume fraction in the symmetry plane for configuration B with the heated windward wall.

The effect of the free convection on the pollutant gas dispersion can be investigated considering the results of $\mathrm{CO} 2$ volume fraction for configuration $\mathrm{B}$ with the heated windward wall of the block, Fig. 7c. The conditions of the wind flow near the heated windward wall give a free convection-to-forced convection ratio $G R / R e^{2}>1.0$. This means that the free convection is expected to have a pronounced effect as indicated by the both results Fig. 7b and 7c. For all distributions shown in Fig. 7, it can be easily noticed that the predictions of the present in-house CFD code are - in general - in good agreement with the predictions of the reference CFD code as well as the corresponding measurements of $\mathrm{CO} 2$ volume fraction.

\subsection{Estimation of code calculation and experimental errors}

An indicator of the code calculation accuracy is identified by the errors in the code calculation as compared with the exact solution. Because of the absence of exact solutions of 
the present test cases, the accuracy indicator can be simply expressed as the overall average value of relative deviations of the present in-house CFD code calculations from those obtained by the reference CFD code, ANSYS 16. These relative deviations were estimated using all significant numerical values of the CDF code predictions discussed above and presented in Figs. 4, 6 and 7. The estimated average values of procedure for the relative deviations for individual flow characteristics are: $3.2 \%$ for the longitudinal velocity component $(\mathrm{u}), 2.6 \%$ for the vertical velocity component $(\mathrm{w}), 15 \%$ for the total flow velocity (U), $2.2 \%$ for the flow temperature $(\mathrm{T})$, and $3.4 \%$ for the gas volume fraction $\left(Y_{v, \mathrm{CO} 2}\right)$. A weighted mean of the all individual average values was calculated giving an overall average value of the relative deviations of $4.8 \%$. This value is small enough such that it can be stated the calculation accuracy of the present in-house CFD code is acceptable.

Concerning the experimental results, an indicator for the uncertainty in the measured value of each flow property is identified by the corresponding experimental errors associated with measuring devices, measuring procedure, and flow conditions. Such uncertainty indicator can be estimated as the ratio between the sum of all possible experimental errors and a reference measured value of the considered flow property. The estimated average uncertainty values of different flow characteristics are: $24.8 \%$ for the flow velocity, $8 \%$ for the flow temperature, and $8 \%$ for the gas volume fraction.

\subsection{The validation process}

The above discussion on the results for the test cases investigated in the present work, shows an overall good agreement with those of the reference CFD code. This agreement gives emphasis on the code correctness for the present in-house CFD code. The code correctness together with the acceptable code calculation accuracy as described above support the decision on the present code verification. This decision with the partial code verification obtained previously by the authors [29], strengthen the claim on the satisfaction of the verification prerequisite condition to begin the validation process.

The first stage of the validation process has been already satisfied via the good qualitative agreement between the predictions of present in-house CFD code and the corresponding flow measurements, as discussed above. The second stage is to examine the discrepancies of the code predictions relative to the flow measurements. These discrepancies can be expressed as the values of the relative deviations of the present inhouse CFD code calculations from the corresponding flow measurements. An appropriate indicator (metric) of the discrepancies associated with a specified flow property is estimated as the global relative deviation that takes into account the entire vertical distribution of this property. The estimation procedure yielded indicator values expressing the code predictions discrepancies of: $9.7 \%$ for the flow velocity, $5.44 \%$ for the flow temperature, and $3.9 \%$ for the gas volume fraction. These discrepancies indicator values are lower than the corresponding experimental uncertainties stated in subsection 5.4. This result is sufficiently acceptable as a criterion to consider good quantitative agreement between the predictions of present in-house CFD code and the corresponding flow measurements. Finally, the qualitative and quantitative agreements with the flow measurements are considered satisfactory to decide on the successful validation of the present in-house CFD code 
Walid J. Al-Nahari et al., Development and validation of an in-house cfd code for ..............

\section{Conclusions}

This paper presents the development of an accurate and efficient semi-implicit pressurebased algorithm with in-house CFD code for Environmental Flow Simulation. The algorithm solves Navier-Stock equations, and conservation equations of energy and mass species, governing gases pollutant dispersion in urban atmospheres. A standard validation process was applied to the present in-house CFD code, using six generic test cases. The test cases simulate a steady-state environmental flow associated with gas flow from a ground point source under different flow conditions. As prerequisite, the application of a standard verification process including the code correctness and calculation accuracy was considered. The prerequisite of the standard verification process has been satisfied through the good agreement of the predictions of the present in-house CFD code with the corresponding predictions of a reference CFD code, ANSYS Fluent 16. The detailed standard validation process was performed by comparing the predictions of the present inhouse CFD code with the corresponding flow measurements especially conducted for the present work in the test section of an Atmospheric Boundary Layer Wind Tunnel facility. The measurements cover different conditions of the flow properties including the volume fraction of the gas pollutant. The predictions of the present in-house CFD code for all generic test cases showed good qualitative and quantitative agreements with the corresponding flow measurements. These agreements establish a satisfactory and successful validation of the present in-house CFD code.

\section{REFERENCES}

[1] Barbosa, P. H. A., M. Cataldi and A. P. S. Freire (2002), "Wind Tunnel Simulation of Atmospheric Boundary Layer Flows", Journal of the Brazilian Society of Mechanical Sciences, 24(3), pp. 177-185.

[2] Wei, L. W., Desmond, L. C., 2005. Atmospheric boundary layer wind tunnel design. TEC group in the University of Adelaide, Australia, 169-174.

[3] Chavez, M., B. Hajra, T. Stathopoulos and A. Bahloul (2011), "Near-Field Pollutant Dispersion in the Built Environment by CFD and Wind Tunnel Simulations", Journal of Wind Engineering and Industrial Aerodynamics, 99(4), pp. 330-339.

[4] Viskanta, R. and R. W. Bergstrom (1973), "Modeling of the Effects of Pollutants and Dispersion in Urban Atmospheres Part I: Thermal Structure", Journal of Applied Meteorology, 12(6), pp. 901-912.

[5] Yassin, M. F. (2004), "Study on Pollutant Dispersion within Urban Area under Changes of Atmospheric Stability and Wind Direction", Ph. D. Thesis, University of Tokyo.

[6] Bady, M. F. (2008), "Study of Pollutants Dispersion and Wind Ventilation Effectiveness in Urban Areas through CFD Simulations and Wind Tunnel Experiments", Ph. D. Thesis, University of Tokyo.

[7] Al-Nehari, H. A. (2010), "A Study on the Effects of Air Pollutants and Dispersion in Urban Atmospheres", Ph. D. Thesis, University of Assiut.

[8] Barranger, N., T. Ternisien and G. Kallos (2015), "An Intercomparison Study between RAMS and CRES-Flow-NS Models and Evaluation with Wind Tunnel Experimental Data: Toward Improving Atmospheric Modeling for Wind Resource Assessment", Journal of Wind Engineering and Industrial Aerodynamics, 142, pp. 272-288.

[9] Silva, J., F. Marques da Silva, A. Couto and A. Estanqueiro (2015), "A Method to Correct the Flow Distortion of Offshore Wind Data Using CFD Simulation and Experimental Wind Tunnel Tests", Journal of Wind Engineering and Industrial Aerodynamics, 140, pp. 87-94.

[10] An, K., J. C. H. Fung and S. H. L. Yim (2013), "Sensitivity of Inflow Boundary Conditions on Downstream Wind and Turbulence Profiles Through Building Obstacles Using a CFD 
Approach", Journal of Wind Engineering and Industrial Aerodynamics, 115, pp. 137-149.

[11] Calautit, J. K. and B. R. Hughes (2014), "Wind Tunnel and CFD Study of the Natural Ventilation Performance of a Commercial Multi-Directional Wind Tower", Building and Environment, 80, pp. 71-83.

[12] Calautit, J. K., B. R. Hughes and S. S. Shahzad (2015), "CFD and Wind Tunnel Study of the Performance of a Uni-Directional Wind Catcher with Heat Transfer Devices", Renewable Energy, 83, pp. 85-99.

[13]Blocken, B. and Y. Toparlar (2015), "A Following Car Influences Cyclist Drag: CFD Simulations and Wind Tunnel Measurements", Journal of Wind Engineering and Industrial Aerodynamics, 145, pp. 178-186.

[14] Al-Nehari, H. A., A. K. Abdel-Rahman and A. El-Moneim (2009), "Design and Construction of a Wind Tunnel for Environmental Flow Studies", Cairo 11th International Conference on Energy and Environment, Hurghada, Egypt-March, 2009.

[15] Al-Nehari, H. A., A. K. Abdel-Rahman, H. M. Shafey and A. E.-M. Nassib "Characterization of a Low-Speed Wind Tunnel Simulating Urban Atmospheres", Journal of Engineering Sciences, Assiut University, Vol. 38(2), pp. 509-532

[16]AIAA (1998), "Guide for the Verification and Validation of Computational Fluid Dynamics Simulations", AIAA G-077-1998(2002), American Institute of Aeronautics and Astronautics, Reston VA.

[17] Oberkampf, W. L. and F. G. Blottner (1998), "Issues in Computational Fluid Dynamics Code Verification and Validation", AIAA Journal 36(5), pp. 687-695.

[18] Oberkampf, W. L. and T. G. Trucano (2002), "Verification and Validation in Computational Fluid Dynamics", Progress in Aerospace Sciences, 38(3), pp. 209-272.

[19] Oberkampf, W. L., T. G. Trucano and C. Hirsch (2004), "Verification, Validation, and Predictive Capability in Computational Engineering and Physics", Applied Mechanics Reviews, 57(5), pp. 345-384

[20] Oberkampf, W. L. and T. G. Trucano (2008), "Verification and Validation Benchmarks", Nuclear Engineering and Design, 238(3), pp. 716-743.

[21]Stern, F., R. V. Wilson, H. W. Coleman and E. G. Paterson (2001), "Comprehensive Approach to Verification and Validation of CFD Simulations-Part 1: Methodology and Procedures", Journal of Fluids Engineering, 123(4), pp. 793:802

[22] Chan, T. L., G. Dong, C. W. Leung, C. S. Cheung and W. T. Hung (2002), "Validation of a Two-Dimensional Pollutant Dispersion Model in an Isolated Street Canyon", Atmospheric Environment ,36(5), pp. 861-872.

[23] Endalew, A. M., M. Hertog, M. A. Delele, K. Baetens, T. Persoons, M. Baelmans, H. Ramon, B. M. Nicolaï and P. Verboven (2009), "CFD Modelling and Wind Tunnel Validation of Airflow Through Plant Canopies Using 3D Canopy Architecture", International Journal of Heat and Fluid Flow, 30(2), pp. 356-368.

[24] Gartmann, A., W. Fister, W. Schwanghart and M. D. Müller (2011), "CFD Modelling and Validation of Measured Wind Field Data in a Portable Wind Tunnel", Aeolian Research, 3(3), pp. 315-325.

[25] Allegrini, J., V. Dorer and J. Carmeliet (2014), "Buoyant Flows in Street Canyons: Validation of CFD Simulations with Wind Tunnel Measurements", Building and Environment, 72, pp. 63-74.

[26] Ramponi, R. and B. Blocken (2012), "CFD Simulation of Cross-Ventilation Flow for Different Isolated Building Configurations: Validation with Wind Tunnel Measurements and Analysis of Physical and Numerical Diffusion Effects", Journal of Wind Engineering and Industrial Aerodynamics, 104-106, pp. 408-418.

[27]Rusch, D., L. Blum, A. Moser and T. Roesgen (2008), "Turbulence Model Validation for Fire Simulation by CFD and Experimental Investigation of a Hot Jet in Crossflow", Fire Safety Journal, 43(6), pp. 429-441.

[28] Suard, S., C. Lapuerta, F. Babik and L. Rigollet (2011), "Verification and Validation of a 
Walid J. Al-Nahari et al., Development and validation of an in-house cfd code for

CFD Model for Simulations of Large-Scale Compartment Fires”, Nuclear Engineering and Design, 241(9), pp. 3645-3657.

[29] Al-Nahari, W. J., M. F. El-Dosoky, M. M. Abdelghany and H. M. Shafey (2014), "Development, Verification and Validation of an In-House CFD Code for Weakly Compressible Flow", Journal of Engineering Sciences, Assiut University, 42(5), pp. 1193-1214

[30] Ghia, U., K. N. Ghia and C. T. Shin (1982), "High-Re Solutions for Incompressible Flow Using the Navier-Stokes Equations and a Multigrid Method", Journal of Computational Physics, 48(3), pp. 387-411.

[31] Slater J. W., (2008), "Driven Cavity", Retrieved from http://www.grc.nasa.gov/www/wind/valid/cavity/cavity.html.

[32]Rumsey, C., (2014), "2D Bump-in-Channel Verification Case - Intro Page", Retrieved from http://turbmodels.larc.nasa.gov/bump.html.

[33] Mateescu, D., C. B. Mei and E. Zuppel (2005), "Computational Solutions of Unsteady Confined Viscous Flows with Variable Inflow Velocities for Unsteady Fluid-Structure Interaction Problems", Proceedings of the Symposium of Flow-Induced Vibrations-2005, ASME PVP Conference, Denver, Colorado, July 2005, ASME Paper PVP2005-71777, pp. 1-11.

[34] Schlichting, H., Boundary Layer Theory. $7^{\text {th }}$ ed., McGraw-Hill, New York, 1979.

[35] Novozhilov, V. (2001), "Computational Fluid Dynamics Modeling of Compartment Fires", Progress in Energy and Combustion Science, 27(6), pp. 611-666.

[36]Bird, R. B., W. E. Stewart and E. N. Lightfoot (2002), Transport Phenomena. $2^{\text {nd }}$ ed., John Wiley \& Sons, New York, 2002.

[37]Irwin, H. P. A. H. (1981), "The Design of Spires for Wind Simulation", Journal of Wind Engineering and Industrial Aerodynamics, 7(3), pp.361-366. 


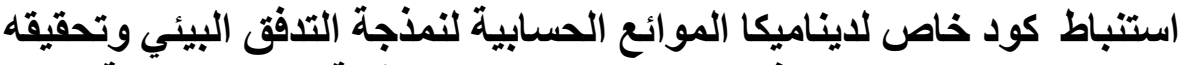

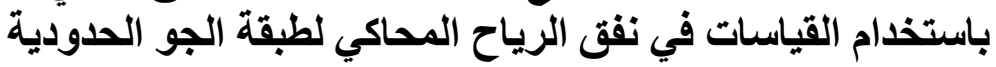

\section{ملخص البحث}

يتعلق هذا البحث بموضوع ذي أهمية وهو نمذجة انتشار الملوثات الغازية خلال التدفق البيئي داخل

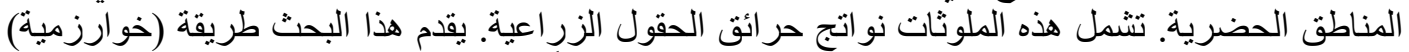

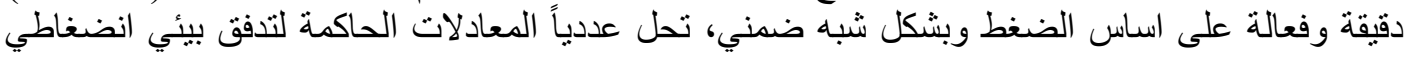

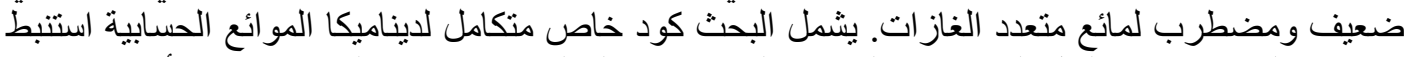

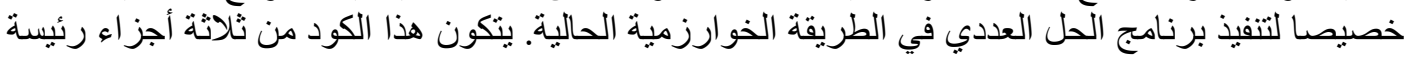

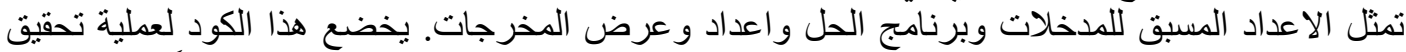

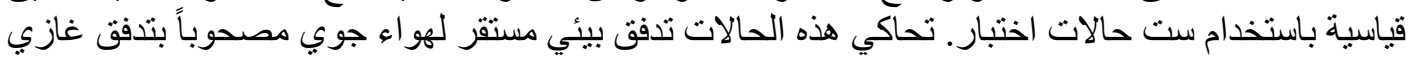

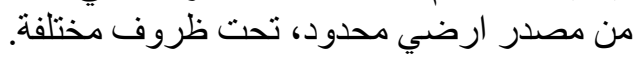

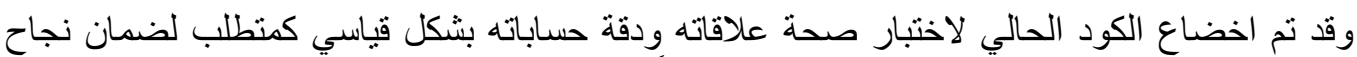

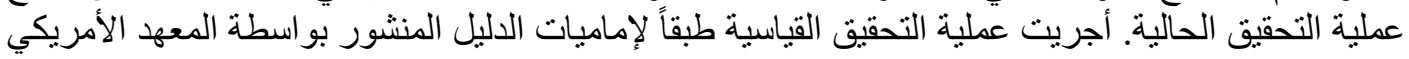

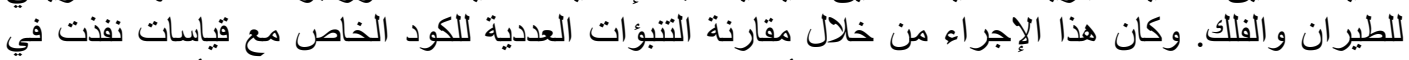

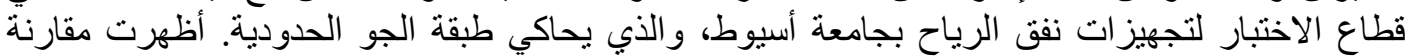

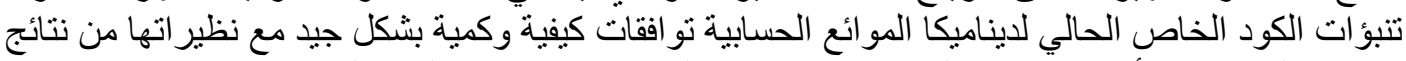

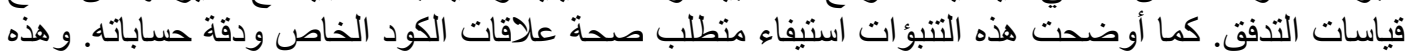

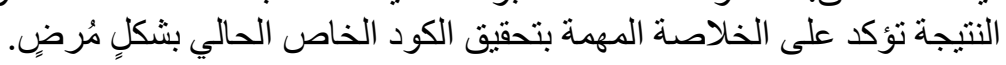

